\title{
Téoros
}

Revue de recherche en tourisme

\section{Déplacements loisirs de semaine dans la région métropolitaine de Montréal en 1982}

\section{Yves Bussière}

Volume 6, numéro 1, mars 1987

Tourisme et transports

URI : https://id.erudit.org/iderudit/1080527ar

DOI : https://doi.org/10.7202/1080527ar

Aller au sommaire du numéro

Éditeur(s)

Université du Québec à Montréal

ISSN

0712-8657 (imprimé)

1923-2705 (numérique)

Découvrir la revue

Citer cet article

Bussière, Y. (1987). Déplacements loisirs de semaine dans la région métropolitaine de Montréal en 1982. Téoros, 6(1), 37-39.

https://doi.org/10.7202/1080527ar d'utilisation que vous pouvez consulter en ligne.

https://apropos.erudit.org/fr/usagers/politique-dutilisation/ 


\title{
Déplacements loisirs de semaine dans la région métropolitaine de Montréal en 1982
}

\author{
par Yves Bussière*
}

Que représentent les déplacements loisirs par rapport à l'ensemble des déplacements faits par la population montréalaise, et quelles en sont les principales caractéristiques? Est-ce que les jeunes ont des modèles de déplacements semblables à ceux de leurs aînés? Quels modes de transport sont les plus utilisés pour les loisirs? Nous fournirons dans ce bref article quelques éléments de réponse à ces questions à l'aide des don= nées de l'enquéte Origine-Destination de 1982 réalisée par la Commission de Transport de la Communauté urbaine de Montréal (CTCUM) ${ }^{11}$. Cette enquête ne recensce que les déplacements réalisés en semaine (du lundi au vendredi). Nous analyserons ici ces déplacements en nous limitant aux déplacements "unidirectionnels" "qui excluent le.retour au domicile. En effet, de façon générale, à chaque déplacement pour un but donné par un mode de transport donne, correspond un retour au domicile, ce qui veut dire, grosso modo, que les volumes de déplacements présentés ici pourraient à toutes fins pratiques être doublés si l'on tenait compte du retour au domicile.

Selon cette enquête, les résidents de la région métropolitaine de Montréal font chaque jour de semaine en moyenne, quelque 2,8 millions de déplacements "unidirectionnels". Comme on peut le voir au tableau 1, le but travail domine nettement avec $39 \%$ des deplacements, viennent ensuite, dans l'ordre, le but étude (18\%), autres buts $(17 \%)$, magasinage $(16 \%)$ et loisirs $(10 \%),{ }^{2}$ Bien que le but loisirs arrive loin derrière les autres buts, il n'en demeure pas moins qu'il représente $10 \%$ des déplacements soit quelque 282,000 déplacements par jour sans compter les retours au domicile. Par habitant, cela représente 0,11 déplacement par jour.

Il est difficile d'évaluer ce que reprếsentent les déplacements loisirs de semaine par rapport à ceux effectués les week-ends. Si $1^{\dagger}$ on se base sur les réponses données lors d'une enquête réalisée sur la R.M.R. de Montréal ${ }^{(3)}$, sur 10 activités de loisirs ${ }^{(4)}$ en moyenne, pour l'année 1981, 24\% des

*Professeur agrege, I.N.R.S.-Urbanisation, Universitt du OuAbec.

répondants déclaraient la semaine comme le moment le plus propice a la pratique de ces activités par rapport à $65 \%$ la fin de semaine. Bien que l'on ne puisse pas extrapoler ces résultats à l'ensemble des activites loisirs ni faire le passage de 5 jours par semaine à 2 jours de week-end, cela indique qu'une bonne proportion d'activités loisirs est faite en semaine, particulièrement les activités qui ne nécessitent pas de quitter la ville, comme la randonnée à bicyclette qui se pratique surtout en semaine.

\section{TABLEAU 1}

Répartition des déplacements unidirectionnels journaliers de semaine par buts, RMR de Montréal, 1982 en vol. et en $\%$

\begin{tabular}{|l|r|r|}
\hline Buts & \multicolumn{3}{|c|}{ volume } & $\%$ \\
\hline travail & 1079000 & 39 \\
\hline étude & 506000 & 18 \\
\hline magasinage & 437000 & 16 \\
\hline loisirs & 282000 & 10 \\
\hline autres & 483000 & 17 \\
\hline total & 2787000 & 100 \\
\hline
\end{tabular}

\section{Mobilité par sexe}

Dans l'ensemble des déplacements, on observe en général une mobilité un peu plus élevée chez les hommes que chez les femmes. En effet, alors que le nombre de déplacements journaliers chez les hommes est de 1,15 , il n'est que de 0,97 chez les femmes (tableau 2).

Si l'on décompose cette mobilité par âge on voit que cette différence par sexe commence dans les groupes $\mathrm{d}^{+}$agge $20-24$ ans, et elle se maintient par la suite pendant tout le cycle de vie bien que l'ecart diminue aे compter de 65 ans. En fait, si on comparait les comportements de mobilité des deux sexes en se limitant aux gens qui effectuent des déplacements ${ }^{(5)}$ on verrait que les différences par sexe s'estompent. Ceci s'explique en grande partie par le fait que les taux d'activite des femmes sont nettement plus faibles que ceux des hommes (en 1982, au Québec, ils étaient de $74,2 \%$ pour les hommes et de $45,9 \%$ pour les femmes ${ }^{(6)}$ ) ce qui explique en grande partie la mobilité plus faible des hommes et des femmes, alors que par rapport aux personnes mobiles (c'est-ă-dire celles qui ont fait au moins un déplacement) les différences par sexe s'estompent.

Qu'en est-il des déplacements loisirs? On pourrait s'attendre au départ à ce que les différences par sexe soient plus faibles que pour les déplacements totaux puisque ce biais dû aux taux d'activité ne joue probablement plus dans le cas des loisirs. En fait, il n'en est rien, et l'écart de $19 \%$ observé pour les déplacements totaux se maintient dans le cas des déplacements loisirs, comme on peut le voir au tableau 3.

Il n'en demeure pas moins que les deplacements loisirs gardent une proportion très stable dans les déplacements totaux. Cette proportion demeure à $10 \%$ chez les femmes comme chez les hommes. Il semblerait donc que la mobilité loisirs ait tendance à suivre celle de la mobilité générale et une éventuelle augmentation de la mobilité des femmes suite a une hausse de leur taux d'activité pourrait vraisemblablement se traduire par une mobilité loisirs accrue.

\section{Déplacements par âge}

Si l'on reprend I'analyse de la mobilité loisirs par âge, on observe une grande variabilité dans les comportements, comme on peut le voir au graphique 1. Alors que la mobilité loisirs moyenne ( 2 sexes réunis) est de 0,11 déplacement par jour, elle est
TABLEAU 2

Déplacements unidirectionnels totaux de la population de 5 ans et plus, par sexe, RMR de Montréal, 1982

\begin{tabular}{|l|c|c|c|}
\hline Sexe & population & déplacements & dépl./hab. \\
\hline hommes & 1266825 & 1463000 & 1,15 \\
\hline femmes & 1363959 & 1324000 & 0,97 \\
\hline total & 2630784 & 2787000 & 1,06 \\
\hline
\end{tabular}




\section{GRAPHIQUE 1}

Par age et sexe, RMR Mti 1982

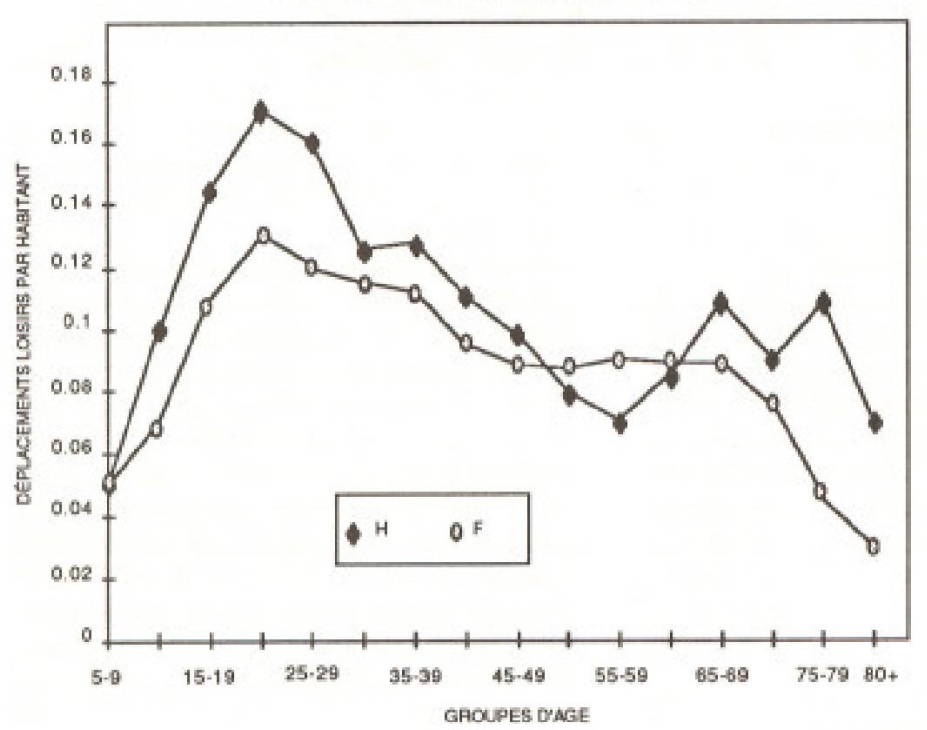

GRAPHIQUE 3

Par age et sexe, RMR Mtl 1982

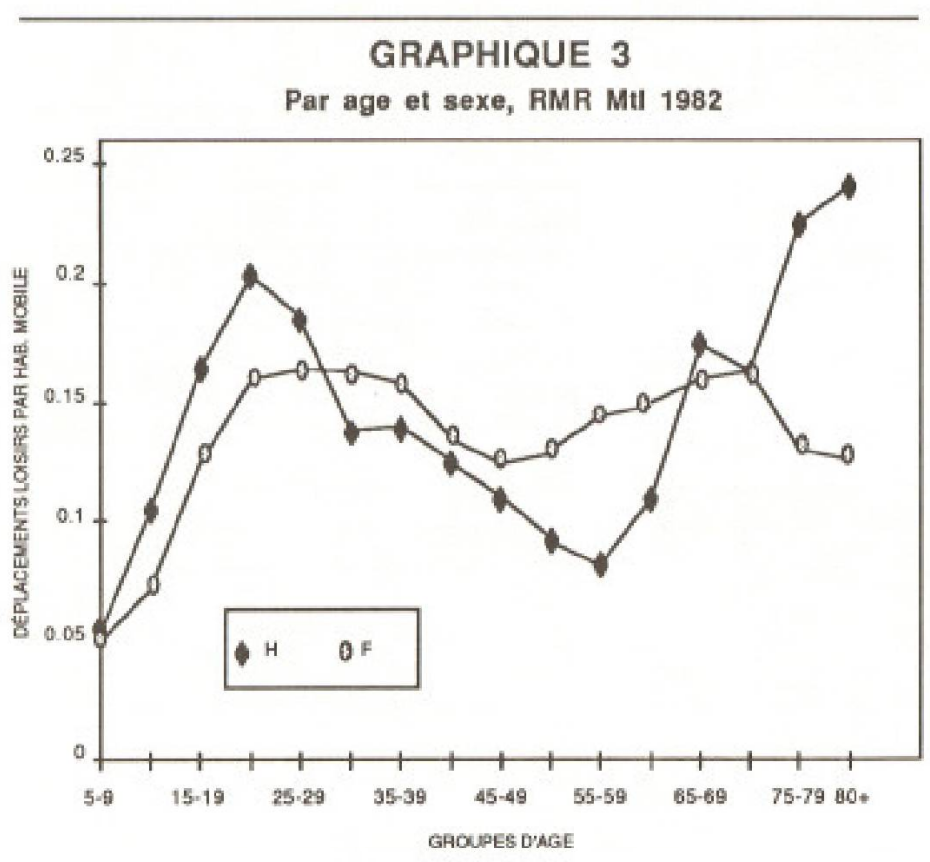

GRAPHIQUE 2

Par age et sexe, RMR Mt! 1982

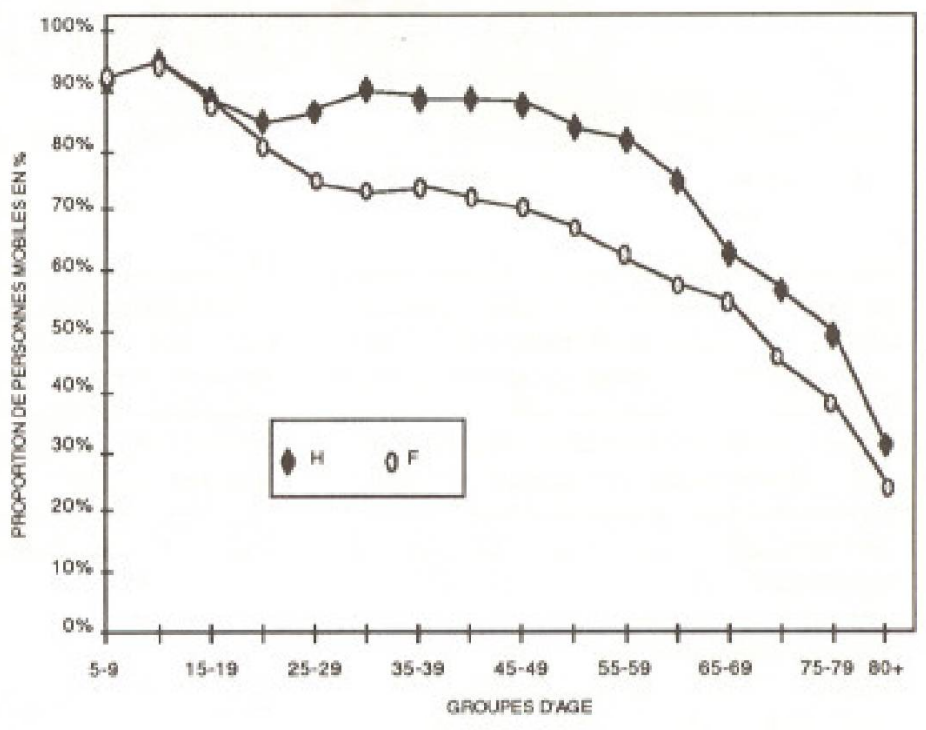

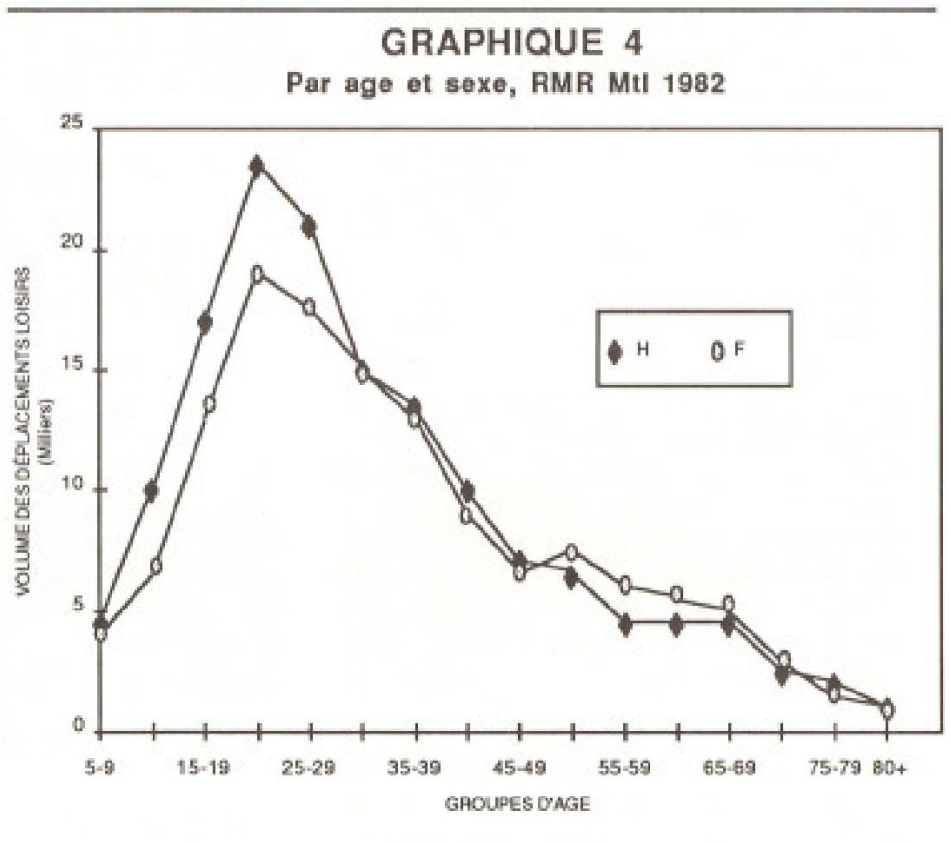


TABLEAU 3

Déplacements unidirectionnels, but loisirs, de la population de 5 ans et plus, par sexe, RMR de Montréal, 1982

\begin{tabular}{|l|c|c|}
\hline Sexé & déplacements & dépl./hab. \\
\hline hommes & 148000 & 0,117 \\
\hline femmes & 134000 & 0,098 \\
\hline total & 282000 & 0,107 \\
\hline
\end{tabular}

beaucoup plus élevée chez les jeunes que chez leurs ainés et elle est nettement plus forte chez les jeunes de 15 à 30 ans, avec un sommet de 0,17 chez les hommes de 20-24 ans. On retrouve donc ici un modèle semblable à celui de la mobilité générale où la mobilité est nettement plus élevée chez les jeunes, avec cependant une différence notable, à savoir une hausse de la mobilité loisirs à compter de 60 ans chez les hommes, et un maintien de la mobilité loisirs chez les femmes à compter de 45 ans. Ce n'est que dans les strates d'âge très avancées que la mobilité loisirs recommence à diminuer, soit après 75 ans chez les femmes et après 80 ans chez les hommes!

Cet indice est évidemment le reflet de deux phénomènes distincts, d'une part, la mobilitế générale des individus et, d'autre part, la décision de se déplacer pour le but loisirs. Compte tenu du fait que la proportion de mobiles, c'est-à-dire de gens qui ont effectué au moins un déplacement dans une journée type, diminue sensiblement avec l'age (graphique 2). Ainsi, si l'on calcule un indice de mobilité loisirs sur la population mobile (graphique 3), on observe une augmentation considérable de la mobilité loisirs chez les jeunes. Pour les hommes, celle-ci atteint un premier sommet de 0,20 déplacement par jour chez les jeunes de 20-24 ans, descend par la suite jusqu'au groupe d'âge $55-59$ ans pour atteindre ensuite un nouveau sommet chez les 80 ans et plus. La mobilité loisirs des femmes mobiles est également étroitement liếe à l'âge, et elle est supérieure à celle des hommes dans les groupes d'âge de 30-35 ans et à 60-64 ans.
Évidemment, en termes de volume, cette traduit pas nécessairement par des volumes de déplacements très élevés compte tenu des baisses d'effectifs dans les cohortes d'âges avancés. Ainsi, à titre d'exemple, dans le groupe d'âge des 80 ans et plus, chez les hommes, la très forte propension de déplacements loisirs des personnes mobiles, de 0,24 , ne correspond qu'à 834 déplacements journaliers pour l'ensemble de la R.M.R. de Montréal, alors que chez les hommes de 20-24 ans, un indice de 0,20 correspond à quelque 24000 déplacements. La demande de transport, en termes d'effectifs, est done le reflet de cette double réalité: d'une part, le nombre de personnes susceptibles de se déplacer que l'on peut représenter par des populations par groupes d'âge, et, d'autre part, des comportements de déplacements. L'effet conjugué de ces différents éléments donne les déplacements effectivement réalisés, que l'on peut représenter en volume, pour les loisirs, par le graphique 4.

\section{Déplacements par mode}

Quels sont les modes de transport les plus utilisés pour les déplacements loisirs? Sontils les mêmes que pour l'ensemble des déplacements? Comme on peut le voir au tableau 4, pour les deux sexes, l'automobile domine avec $58 \%$ des déplacements comparativement à $24 \%$ pour les transports en commun et $19 \%$ pour les autres modes.

Pour les loisirs, la proportion de déplacements par automobile passe à $69 \%$ au détriment des transports en commun ( $18 \%$ ) et des autres modes (13\%). Si l'on fait les mêmes comparaisons par sexe, on observe forte propension des personnes âgées ne se que la propension à utiliser l'automobile est beaucoup plus forte chez les hommes que chez les femmes ( $65 \%$ vs $50 \%$ ) pour l'ensemble des déplacements. Au niveau des déplacements loisirs, ces différences s'estompent considérablement avec $71 \%$ des déplacements en automobile pour les hommes comparativement à $67 \%$ pour les femmes.

Ceci n'a cependant guère d'impact sur la congestion urbaine puisque seulement $2,1 \%$ des déplacements loisirs sont faits pendant la période de pointe du matin (7h à 9h). Cette proportion varie peu selon le sexe $(1,9 \%$ chez les hommes et $2,2 \%$ chez les femmes). Ces déplacements ne constituent que $0,5 \%$ des déplacements de pointe du matin; ils constituent, par ailleurs, $16 \%$ des déplacements hors-pointe.

\section{Conclusion}

La demande de déplacements loisirs en semaine demeure un phénomène important; surtout effectués en automobile, ces déplacements sont faits principalement horspointe et devraient avoir à priori aucun impact sur la congestion urbaine des heures de pointe de semaine. Il n'en demeure pas moins que $16 \%$ des déplacments horspointe sont des déplacement ayant pour but les loisirs, et que $18 \%$ de ces déplacements sont faits par les transports en commun. À moyen terme, si des modifications dans les habitudes de vie venaient augmenter la part des déplacements loisirs, il pourrait devenir important de pousser davantage ce genre d'analyse en tenant compte d'autres variables telles que le taux d'activité des femmes, la structure des ménages ou encore la localisation résidentielle, afin d'en évaluer l'impact sur la demande de transport. $f$

TABLEAU 4

Principaux choix de mode pour les déplacements tous buts et but loisirs, par sexe, R.M.R. de Montréal, 1982 en \%

\begin{tabular}{|l|l|l|}
\hline Mode & tous buts & but loisirs \\
\hline \multicolumn{3}{|l|}{ 2 sexes } \\
\hline Transp. en commun & 24 & 18 \\
\hline automobile & 58 & 69 \\
\hline autres modes & 19 & 13 \\
\hline \multicolumn{2}{|c|}{ hommes } \\
\hline Transp. en commun & 19 & 16 \\
\hline automobile & 65 & 71 \\
\hline autres modes & 16 & 13 \\
\hline \multicolumn{2}{|c|}{ femmes } \\
\hline Transp. en commun & 29 & 20 \\
\hline automobile & 50 & 67 \\
\hline autres modes & 21 & 13 \\
\hline
\end{tabular}

